\title{
Passive Maple-seed Robotic Fliers for Education, Research and Entrepreneurship
}

\author{
D.M. Aslam, A. Abu-Ageel, M. Alfatlawi, M.W. Varney, C.M. Thompson \& S.K. Aslam \\ College of Engineering, Michigan State University, Michigan, USA \\ Correspondence: Dean Aslam, College of Engineering, Michigan State University, East Lansing, MI 48824, USA.
}

Received: January 14, $2014 \quad$ Accepted: February 5, $2014 \quad$ Online Published: March 10, 2014
$\begin{aligned} & \text { doi:10.1114/jets.v2i2.318 } \\ & \text { URL: http://dx.doi.org/10.11114/jets.v2i2.318 }\end{aligned}$

\begin{abstract}
As inspirations from flora and fauna have led to many advances in modern technology, the concept of drawing ideas from nature for design should be reflected in engineering education. This paper focuses on a maple-seed robotic flier (MRF) with various complexities, a robotic platform modeled after the samaras of maple or ash trees, to teach STEM concepts to K-12 learners. Experiments using MRFs were also designed and incorporated into an undergraduate engineering course. Details are given on how the MRFs have been incorporated into K-12 camps and the undergraduate course. Unique features of MRF development are (a) very simple (using LEGOs and paper) to extremely complex (incorporation of microcontrollers and sensors) learning suitable for pre-K children, free-will adult learners, workforce training, undergraduate and graduate students, (b) research-oriented education, (c) entrepreneurship education and (d) trans-disciplinary education and research. Using microfabrication and nanofabrication technologies, the long range goal is to mass produce MRFs for surveillance in hard-to-reach and dangerous environments.
\end{abstract}

Keywords: STEM, robots-fliers, embedded-intelligence, programming, education, entrepreneurship

\section{Introduction}

Recent advances in sensors and microsystems have led to fascinating developments in man-made and nature-made robotic systems that can be used for education and outreach. Interesting examples include humanoid robots (Kosuge, 2008) and genetically modified E. Coli producing aspartame, insulin, and ethanol (Olson, et al., 2007). Although, a number of robotic devices and systems have been introduced and marketed for educational use, two robotic systems introduced by LEGO Mindstorms have fascinated the robot-inspired learners; these are RCX and NXT programmable robots first marketed in 1999 and 2006, respectively (Beck, et al., 2004). One author of this paper (D. Aslam) has, starting with the RCX robot, developed a series of evolving robotic learning modules that led to the theme of this paper. Some examples of new concepts for such learning modules include the Robot Inspired Learning System (RILS) (Aslam, et al., 2003), Technology Assisted Science, Engineering and Math (TASEM) (Varney, et al., 2012; Aslam, et al., 2008; Aslam, et al., 2006), and more recently Functionalized Bricks with Embedded Intelligence (FBEI). A typical example of a FBEI is a passive Maple-seed Robotic Flyer (MRF) as described in this paper (Figure 1).

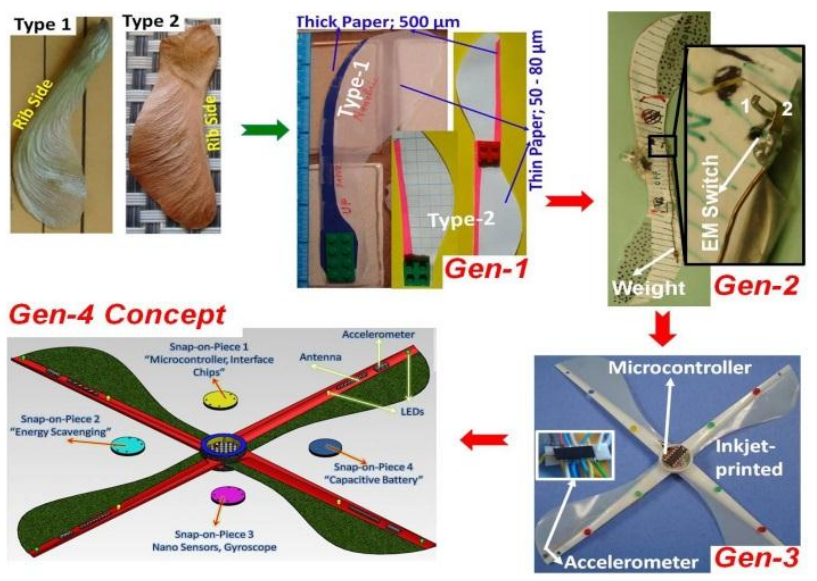

Figure 1. MRF development for education, research and entrepreneurship. 


\section{Maple-seed Robotic Flyer (MRF)}

Traditionally, nature-inspired engineering systems have been of great interest in a number of areas (Thakoor, 2004). For example, active (Ulrich, 2010; Jameson, et al., 2010) and passive (Harned, 1997; Aslam, et al., 2010; Alfatlawi, et al., 2012) MRFs have recently been demonstrated using the underlying concepts of nature-made maple samaras. Since robotics education enhances skills in the areas of critical thinking, inquiry, and systems, a number of studies have focused on the use of robotics for science, technology and engineering (Sullivan, 2008). This paper reports the design, fabrication and testing of MRFs for (a) a very-simple to extremely-complex learning suitable for pre-K children, free-will adult learners, workforce training, undergraduate and graduate students, (b) research-oriented education, (c) entrepreneurship education and (d) trans-disciplinary education and research. Early introduction into the applied sciences in innovative ways can increase student motivation and interest in developing areas of study today. Robotics being a trans-disciplinary field incorporates mathematics, computer sciences, electronics, physics, and material science (Jimenez Jojoa, 2010). Experiments reported in this study were used to teach STEM concepts to K-12 learners and were incorporated into an undergraduate engineering course.
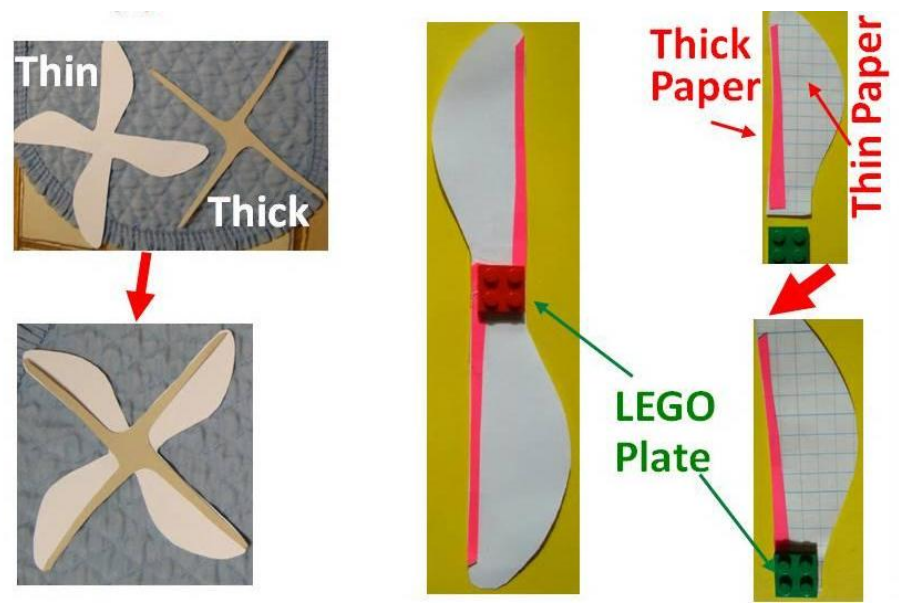

Figure 2. Fabrication of 1, 2 and 4 wing MRFs using paper and LEGO pieces.

Generation one MRFs (Gen-1), modeled after two shapes of maple seeds found in nature, are very simple and contain no sensors or control electronics, as shown in Figure 2. Gen-2 MRFs have simple circuits and batteries. Inkjet-printed Gen-3 MRFs have very simple to very complex control electronics, sensors, LEDs, wireless interfaces and batteries. Gen-4 MRFs are mass-producible and have sophisticated control electronics (including a microcontroller), sensors, actuators (for flight control), wireless interfaces, energy scavenging devices, capacitor batteries, and multimedia devices. The long-term goal is to mass produce MRFs for surveillance in hard-to-reach and dangerous environments using microfabricaton and nanofabrication technologies.

\subsection{Generation One (Gen -1) MRF}

Gen-1 MRF exploration starts with a simple but interesting question: whether students can replicate the technology of the maple-seed flyers found in nature using paper and LEGO pieces? Through a series of design experiences students generated examples similar to Figure 2, using LEGO plates as a "seed", attached to paper wings (the focus has been on wings with straight ribs). The design parameters in this very simple MRF are materials (wing, rib, and seed), shape, size, number of wings, and other such things. Such a large range and complexity of design parameters allows the use of this simple activity for different grade levels and for learners of different interest levels and backgrounds. The National Science Education Standards (NSES) emphasize the ability to use inquiry in science at all grade levels.

Gen-1 allows for the simplicity of basic observation skill development in early elementary on to more complex inquiry experiment development in secondary education. The NSES outline four goals for school science. Two of these goals are showcased within Gen-1 MRF: excite learners and explore a particular parameter in greater depth. A parameter of interest for learners to explore is the weight that a one or multiple wing MRF can carry. This can be investigated by attaching more LEGO bricks to the backend of the MRF. This study is important to learners so they can intelligently integrate more components on the MRF. Figure 3 shows the maximum weight that MRFs of different wing numbers and sizes can carry. 


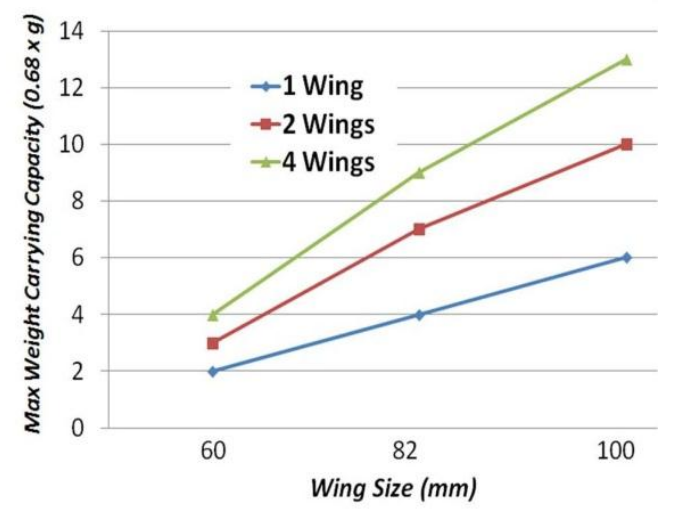

Figure 3. Weight-carrying capacity of Gen-1 MRFs

\subsection{Generation Two (Gen -2) MRF}

Gen-2 MRF learning activities, moving toward preliminary system integration, are explored by adding an on-board simple electronic circuit containing an electromechanical switch, a light emitting diode (LED) and a battery (Figure 4). The circuit is designed such that the LED lights up only during the flight of the MRF. The actuation of the switch by centrifugal force (force directed away from the center) makes the Gen-2 MRF an electromechanical system (EMS) that is a large, simplified version of MEMS or microsystems. Therefore, Gen-2 activities are a fun way of learning about microsystems and nanosystems.



Figure 4. Battery assembly and circuit for Gen-2 MRF.

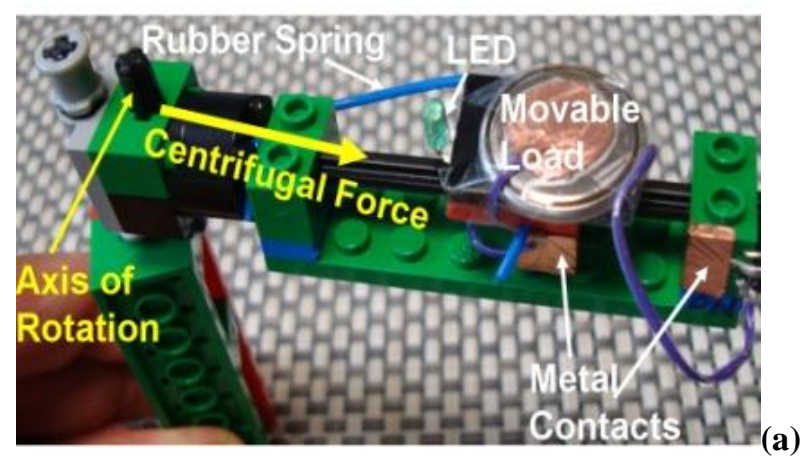




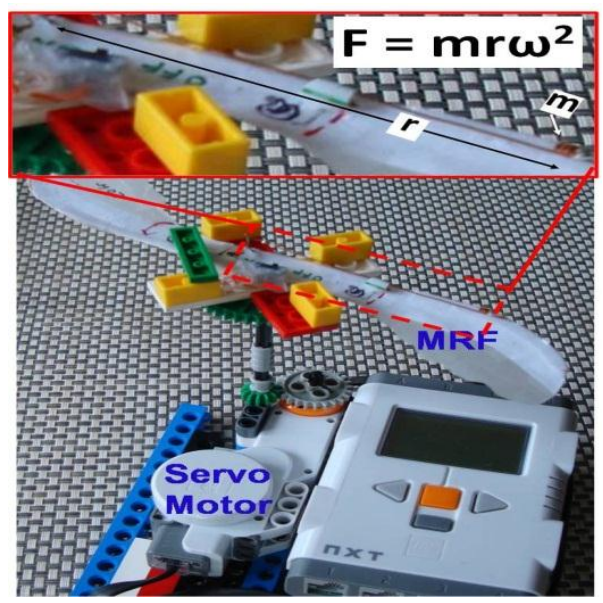

(b)

Figure 5. Models to (a) explain the operation of the EM switch and (b) measure RPM of the MRF at which the switch turns on.

To build an on-board EMS circuit, a very simple technique was developed to connect wires to the two sides of a regular button-cell battery using stripped wires and copper tape as shown in Figure 4. Figure 4 also shows a schematic of an EMS circuit that was incorporated on a 2-wing, type-1 MRF. A switch was made using a thin metal strip, labeled as 2 on the lower right side of Figure 4, and a fixed metal pin, which is labeled as 1. When the MRF is in flight, a weight attached to the metal strip pulls outward due to centrifugal force and connects terminals 1 and 2, completing the circuit; this causes the LED to illuminate.

When the Gen-2 MRFs were shown to summer camp learners and to national and international learners in outreach presentations during 2010 and 2012, they asked a number of questions. "How does the electromechanical switch work?" "How many RPM [revolutions per minute] are needed for the switch to turn on?" "How can one measure RPM?" "What else can one integrate onto the MRF?" "Can the MRFs communicate with one another?" "Can they sense environmental factors?"

To explain the operation of the electromechanical switch, a module was built using a battery, a LED, and a switch. The battery and LED unit, shown in Figure 5(a), was mounted on a rotating LEGO construct. When the LEGO arm spins about the axis of rotation, the unit moves away from the center connecting the two metal electrodes (made of copper tape), lighting the LED when the RPM exceed a certain value. This LEGO device, which can be easily built by the learners, helps to explain the operation principle of the switch in an interesting way.

As shown in Figure 5(b), a commercially available NXT LEGO robot can measure the RPM of its motor with an affixed MRF or the RPM of the device shown in Figure 5(a), and therefore, can find the RPM at which the switch turns on. Sample LEGO programming code to measure the RPM is provided in Figure 6. Once the RPM are measured, the centripetal acceleration (Figure 6) and centrifugal force can be computed using the formula:

$$
\mathrm{F}=\mathrm{mr} \omega^{2}
$$

where $\omega$ is angular velocity.

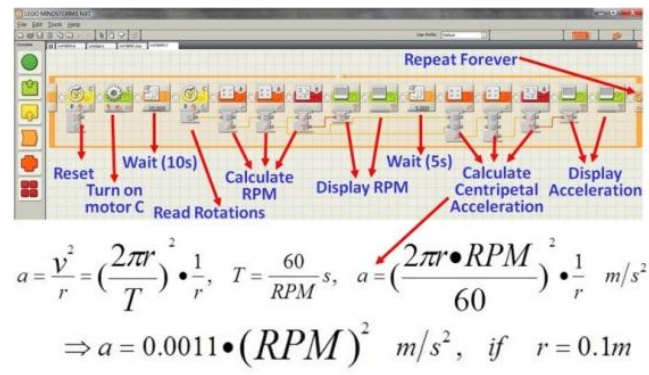

Figure 6. LEGO Mindstorms programming code and equations used for RPM measurement.

Based on activities in summer camps, during 2010 and 2011, children in K-2 grades were most interested in making Gen-1 MRFs and playing with multi-wing MRFs with LEDs, whereas the students in higher grades were interested in making Gen-2 MRFs with on-board electronics that enable the LEDs only during MRF flight. While levels of engagement with the activity were obviously varied due to ages and capabilities of the two groups of students, the 
contribution to inquiry development were highlights of this experience. Due to their importance to development of science inquiry skills, students must be provided with opportunities to use science ideas and engage with the discourses of science (Krajicik et al., 2010). At both levels, students were very involved with the science of their MRFs and had to go beyond critical thinking to discourse of scientific ideas, and eventually applied those concepts to the MRF.

These technology-assisted design activities demonstrate several learning concepts that could be used in MRF curriculum. The practical coursework brings an innovative approach and creative opportunities to learning and allows students to add a research component to their studies. This research component bridges the gap from course material to practical application in the laboratory through innovative ideas (Singh, et al., 2006). This helps clarify important curriculum points for undergraduate students. The learning objective, often ignored in robotics laboratories (Feisel, et al., 2005), is to understand the maple flyer and then think critically to incorporate their knowledge and ideas to push the flyer to its technological limits.

The learner creativity is enhanced when they start thinking critically about the design options that are possible for building advanced MRFs, which could include sensors or a wireless interface. The teachers and students alike are learning and exploring core ideas in STEM that cut across multiple disciplines. The students encounter ideas about system integration (science, engineering, technology), flight dynamics (science, engineering, math), revolutions per minute (science, math, engineering, technology), LEDs (science, engineering, technology), Centrifugal Force (science, math), spring constant (science, engineering, technology) of the EM switch (engineering, technology), design options (Gen-1 to Gen-4 MRFs), their weight carrying ability (technology, engineering), inter MRF communication (engineering, technology) and data exchange (engineering, math, technology), etc. The Gen-3 study, addresses advanced system integration concepts, which help to understand the technologies used in microsystems and nanosystems.

\subsection{Generation Three (Gen -3) MRF}

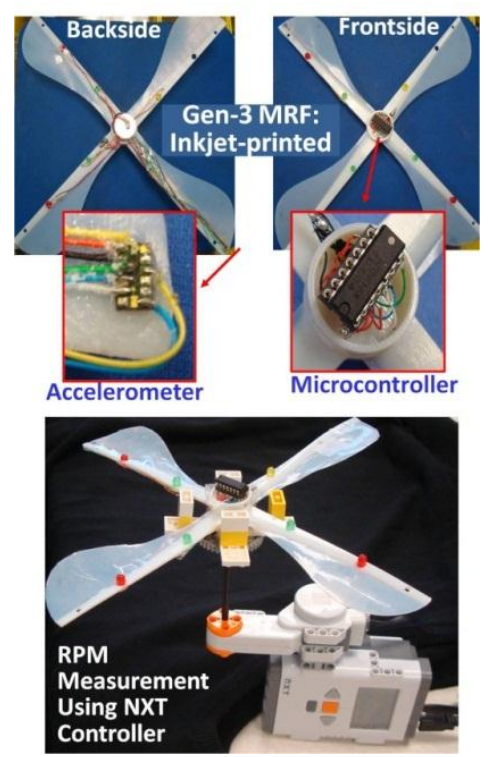

Figure 7. Gen-3 MRF manufactured using an inkjet printer (above) and RPM measurement (below).

The Gen-3 MRF activity was of great interest to freshmen in an undergraduate course. The students were excited about MRFs with sensors, microcontrollers, wireless interfaces and batteries. The Gen-3 activity is scalable to different levels of difficulty for students in different grades. Different design options related to wireless interfaces, sensors, energy sources (batteries versus capacitors versus energy harvesting), and packaging can be explored. The learning activities scale-up, with the knowledge, grade, and investment by the teachers and students. True change happens when teachers are trained in all the related concepts involved in system integration.

The study of Gen-3 MRFs, a step towards mass production of MRFs, starts with inkjet-printing a complete MRF with placeholders for control electronics, sensors, and a battery. Since the 3D inkjet technology for metal interconnects is not available yet (Feisel, et al., 2005; Sanchez-Romaguera, et al., 2008), a commercial inkjet printer (Objet Connex350 ${ }^{\mathrm{TM}}$ ), allowing different plastic materials, was used. A fully printed Gen-3 MRF is shown in Figure 7. The main goal of this generation is to collect, store, and use data from the surrounding environment. The information stored in on-board memory can be wirelessly transmitted to a central location for analysis of the environments that the MRFs have 
monitored. The current version of the Gen-3 system (Figure 7) has only two types of sensors: a temperature sensor and a three-axis acceleration sensor (Feisel, et al., 2005; Foerster, et al., 1999) that can be used to monitor revolutions per minute (RPM), speed of wind, time of flight and other parameters.

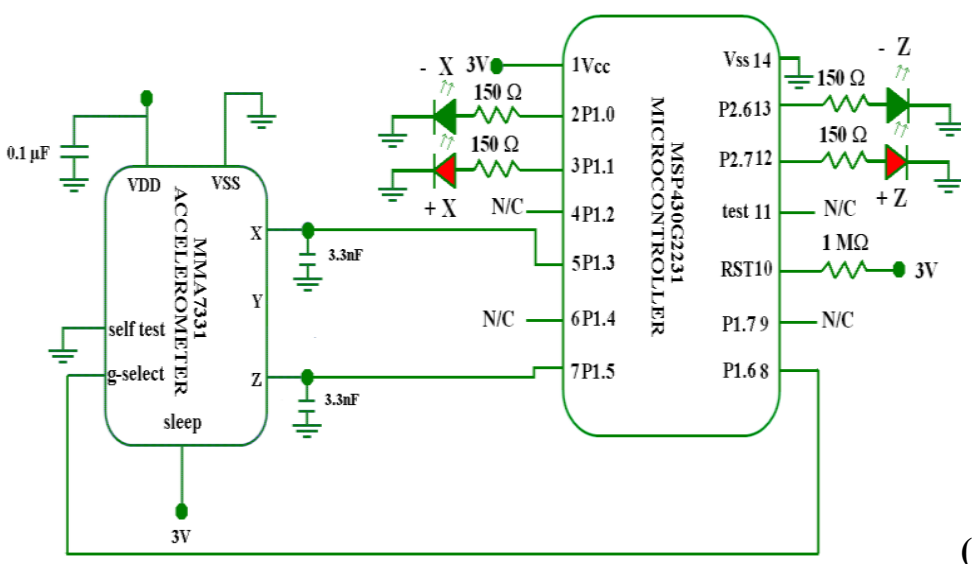

(a)

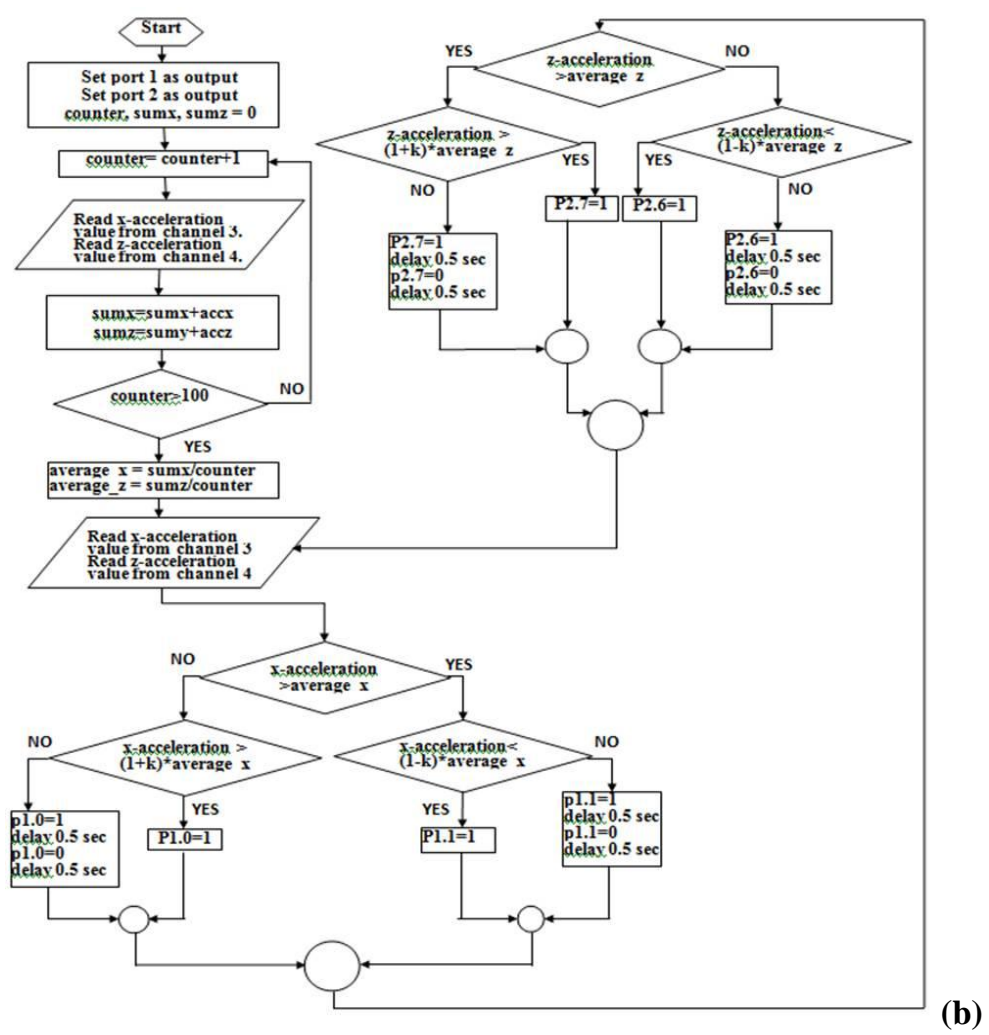

Figure 8. Circuit diagram for Gen-3 MRF (a) and C-code flow diagram (b).

Texas Instrument's MSP430G2231 microcontroller is used as the brain of Gen-3 MRF, which was selected due to its (a) low power consumption (active mode uses $220 \mu \mathrm{A}$, standby mode uses $0.5 \mu \mathrm{A}$, and off mode uses $0.1 \mathrm{uA}$, for supply voltage [VCC] between $1.8 \mathrm{~V}$ and $3 \mathrm{~V}$ ), (b) very low cost for the programmer, C-compiler and microcontroller (all under \$5), (c) 16-bit RISC architecture, (d) high-resolution 10-bit successive-approximation analog-to-digital converter (ADC), (e) reasonably large flash memory of 128 Bytes, and (f) on-board temperature sensor. Other options from the MSP430 microcontroller family are available for higher resolution measurements, such as the MSP430F2013 with a 16-bit Sigma-Delta ADC.

The circuit diagram shown in Figure 8 (a) was incorporated on the Gen-3 MRF to accomplish the following tasks:

a. Direction Detection 
Table 1. X \& Z Axes Output Voltage

\begin{tabular}{ccc}
\hline \multicolumn{2}{c}{$\mathrm{V}_{\mathrm{CC}}=3 \mathrm{~V}$, sensitivity $=0.308 \mathrm{Vg}^{-1}$} \\
\cline { 2 - 3 } Acceleration & \multicolumn{2}{c}{ Output Voltage $(\mathrm{V})$} \\
\hline 0 & 1.5 & Z-axis \\
$g$ & 1.808 & 1.808 \\
$4 g$ & 2.113 \\
$-g$ & 1.192 & 3 \\
$-4 g$ & 0.268 & 0.576
\end{tabular}

In order to detect the direction, a low power accelerometer (MMA7331) was used, which senses the positive and negative acceleration in three directions. The sensitivity can be selected for one of two ranges, -4 to 4 times Earth gravity or -12 to 12 times Earth gravity. The device outputs a voltage ranging from $0 \mathrm{~V}$ to $3 \mathrm{~V}$ to indicate the measured accelerations, as shown in Table 1.

The direction detection program, written in $\mathrm{C}$, starts with a calibration (when MRF is not moving), which samples the accelerometer output 100 times and then takes average values of acceleration in the radial and axial directions (X and $\mathrm{Z}$ axes). These values are multiplied by some threshold factors and stored in the microcontroller memory. After the MRF is released from a certain height, the program starts taking acceleration data and comparing them with the average values stored during the calibration phase. If the acceleration in any direction exceeds the stored calibration value for that direction (times some threshold), the red LED lights up. When the acceleration drops below the calibration acceleration threshold, the green LED lights up. The flashing of red LEDs represents clockwise spinning and upward movement, while the flashing of green LEDs represents counter clockwise spinning and downward movement. During testing, the Gen-3 MRF performed as expected. The MRF starts spinning after it has fallen approximately $71.12 \mathrm{~cm}$. An NXT controller (Figure 7) was used to measure the RPM at which the LEDs are switched on at 60.5 RPM and stay on above 67.6 RPM.

\section{b. Temperature Sensing}

The environmental temperature can be calculated according the following equation:

$$
T\left[{ }^{\circ} \mathrm{C}\right]=\left(\frac{V_{\text {Sensor }}-V_{0^{\circ} \mathrm{C}}}{T C_{\text {Sensor }}}\right)-273
$$

where $\mathrm{T}$ is the temperature in Celsius, $\mathrm{TC}_{\text {Sensor }}$ is the sensor temperature coefficient, $\mathrm{V}_{\text {Sensor }}$ is the measured voltage, and $\mathrm{V}_{0^{\circ} \mathrm{C}}$ is the reference voltage for $0^{\circ} \mathrm{C}$. The measured values are stored in volatile memory, but the last value measured remains stored in flash memory.

\subsection{Generation Four (Gen -4) MRF}
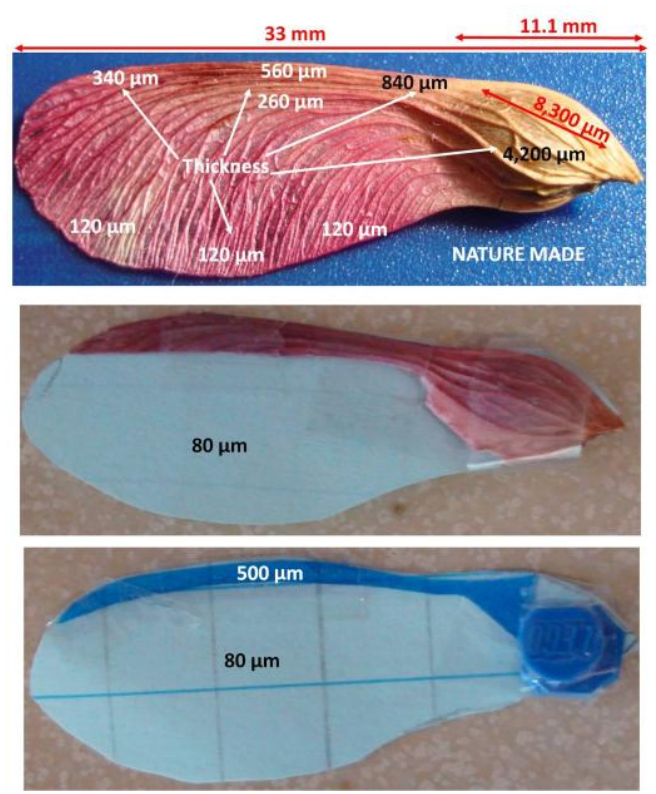

Figure 9. Nature-made maple-seed fliers and manmade MRFs. 
During the fabrication and testing of Gen-3 MRFs, it became obvious that (a) the 3D inkjet-printing does not allow wing thicknesses below $150 \mu \mathrm{m}$ and (b) even at 150- $200 \mu \mathrm{m}$ the wings start curving and bending at room temperature in a few hours. Microfabrication seems to be a better option for smaller MRFs and thinner wings. Whether the Gen-4 MRFs should be a 1-wing, 2-wing, or 4-wing design depends upon (a) their flying characteristics and (b) fabrication technologies that can be used to mass produce inexpensively. The flying characteristics are dependent on the materials (density, stiffness, etc.) used MRF fabrication, wing number and size, total weight of mounted components and surface morphology of wings.

A study focusing on the smallest hand-made one-wing MRF, as seen in Figure 9, indicated that the nature-made flier flew the longest followed by a paper MRF that borrowed the seed and rib from a nature-made flier (middle in Figure 9). The manmade MRF (bottom in Figure 9) flew the shortest. Generally it was also found that the one-wing MRF flies longer than a 2- or 4-wing MRF when other parameters are held proportionately constant. Because a 4-wing MRF drops down faster, it could be used to carry a large number of small microfabricated MRFs that can be launched near the environment that needs to be monitored.

Due to recent advances in fabrication techniques and available fabrication materials for integrated circuits, sensors and MEMS, a number of Gen-4 fabrication options are available. One potential route for fabricating MRFs would be molded plastic. These MRFs could then be assembled with electronics, similar to the Gen-3 MRFs. The cost of injection molding, for mass production, would be significantly lower than the 3D printing used to create Gen-3 MRFs; however the assembly time might still inhibit mass production.

Microfabrication would be useful in MRF mass production due to increased repeatability and throughput. Bulk micromachining techniques, which have been used in industry for the past 50 years (Kovacs, et al., 1998), could be used to create monolithic MRFs with integrated electronics. The depth and size of the wings could be determined using a chemical or electrochemical etch stop in a dissolved wafer process (Gianchandani, et al., 1992). Alternatively, MRFs could be produced using surface micromachining on silicon-on-insulator (SOI) wafers. Potential advantages to using a SOI process include additional device types (e.g. photonics or RF applications) and smaller transistors.

\section{Practicality in Education}

Utilizing questionnaires, undergraduate students who participated in the MRF lab each semester were able to demonstrate the overall educational value of the experience. The questionnaires used a scale between 0 and 10 for students to rate different aspects of the lab experience with 10 being the highest rating. In 2011 there was one undergraduate section completing the MRF lab in both fall and spring semesters while during the second year in 2012 there were two sections in the spring semester. During the first year of the MRF lab, 17 students completed questionnaires rating their individual learning experience, their interest in the experience, the lab design, and the lab experience overall. During the second year of the MRF lab, 28 students completed questionnaires rating the same aspects but with the addition of rating the innovation and fun in the MRF experiment. The data has been compiled into two tables. Table 2 includes the 2011 students' highest and lowest ratings, their average ratings, and the standard deviation of that data set separated by semester and experiment aspect. Table 3 includes the 2012 students' highest and lowest ratings, their average ratings, and the standard deviation of that data set separated by course section and experiment aspect.

Looking first at the overall experiment ratings, the lowest average rating was 7.80 with standard deviation 1.08 from section 1 in 2012 and the highest was 9.46 with standard deviation of 0.81 in spring of 2011. The high student ratings of the overall experiment may be explained by looking at the other experiment aspects rated. The interest level in the experiment was fairly consistent within the first year with averages of 8.5 with standard deviation 1.62 and 9.33 with standard deviation 0.82. With interest levels rated above 8 we can infer that in 2011, the intrinsic motivation to learn in this lab was above average. Interestingly, the level of interest, in both sections, in the second year of the lab was lower than the students' ratings in the first year of the lab. Section 1 on average had an interest level of 6.87 with standard deviation 1.41 versus the 8.07 average rating with standard deviation 1.50 of Section 2. While section 1 interest rating was below 7, it was still above the rating of 5 (which indicates indifference to the lab). This shows the students considered the lab to be above moderately interesting and so both sections may have had a higher intrinsic motivation to learn in the second year much like in the first year.

Furthering the case for higher intrinsic motivation were the scores for rating the learning experience. All average ratings between year one and year two were above 7 and had standard deviations around 1 . Where 5 is a student's typical educational experience, having the learning experience of the MRF lab rated so highly during the first and second years speaks to the lab's educational value to the student. The lab design is incredibly important to the learning experience of the student because a well-developed lab allows for clearer instruction and goals within an activity. In both years, the students rated the lab as very well-developed with no average rating below 8 . This high of a rating every semester and 
section was much appreciated by the lab development staff since the aspects of the experiment were built very deliberately to allow the students the freedom to explore and think critically within the concept of the MRF.

The second year of the lab experience added fun and innovation within the experiment for students to rate. This was an attempt to try and understand if the freedom and exploration highlighted within the lab design was understood and appreciated by the students. Between the two sections in spring of 2012, both rated innovation and fun in experiment very high with ratings of 7.87 with standard deviation 1.55 and 8.35 with standard deviation 1.39 . This leads us to believe that the MRF experiment piqued interest in students not only just through the concept but also because the experiment allowed students to be innovative and make the MRF unique.

What can be gained from all of this information is that the MRF lab has much to offer students. It is well designed so that the innovation and fun within the experiment can catch students' interest and increase their intrinsic motivation to engage in the activity which may contribute to a positive learning experience making the overall experience more valuable.

Table 2. MRF Lab year 1 - 2011

\begin{tabular}{|c|c|c|c|c|c|}
\hline Semester & Number of Participants & Lowest Score & Highest Score & Group Average & Standard Deviation \\
\hline Question asked & \multicolumn{5}{|c|}{ This experiment was a good learning experience for me. } \\
\hline Spring 2011 & 6 & 7 & 10 & 9.17 & 1.17 \\
\hline Fall 2011 & 12 & 7 & 10 & 8.50 & 1.17 \\
\hline Question asked & \multicolumn{5}{|c|}{ My interest in this experience } \\
\hline Spring 2011 & 6 & 8 & 10 & 9.33 & 0.82 \\
\hline Fall 2011 & 12 & 5 & 10 & 8.50 & 1.62 \\
\hline Question asked & \multicolumn{5}{|c|}{ The laboratory experiences are well-designed } \\
\hline Spring 2011 & 6 & 8 & 10 & 9.33 & 0.82 \\
\hline Fall 2011 & 12 & 5 & 10 & 8.25 & 1.48 \\
\hline Question asked & \multicolumn{5}{|c|}{ Overall rating of experiment } \\
\hline Spring 2011 & 6 & 8 & 10 & 9.46 & 0.81 \\
\hline Fall 2011 & 12 & 5 & 10 & 8.35 & 1.11 \\
\hline
\end{tabular}

Table 3. MRF Lab Year 2 - Spring 2012

\begin{tabular}{|c|c|c|c|c|c|}
\hline Section & Number of Participants & Lowest Score & Highest Score & Group Average & Standard Deviation \\
\hline Question asked & \multicolumn{5}{|c|}{ Overall rating of the experiment } \\
\hline Section 1 & 15 & 6 & 10 & 7.80 & 1.08 \\
\hline Section 2 & 13 & 6 & 10 & 8.23 & 1.30 \\
\hline Question asked & \multicolumn{5}{|l|}{ Learning Experience } \\
\hline Section 1 & 15 & 4 & 10 & 7.13 & 1.55 \\
\hline Section 2 & 13 & 6 & 10 & 7.38 & 1.19 \\
\hline Question asked & \multicolumn{5}{|c|}{ My interest in this experience } \\
\hline Section 1 & 15 & 3 & 9 & 6.87 & 1.41 \\
\hline Section 2 & 13 & 5 & 10 & 8.08 & 1.50 \\
\hline Question asked & \multicolumn{5}{|c|}{ Innovation and fun in the experiment } \\
\hline Section 1 & 15 & 5 & 10 & 7.87 & 1.55 \\
\hline Section 2 & 13 & 6 & 10 & 8.38 & 1.39 \\
\hline Question asked & \multicolumn{5}{|c|}{ The activities are well designed } \\
\hline Section 1 & 15 & 6 & 10 & 8.20 & 1.08 \\
\hline Section 2 & 13 & 6 & 10 & 8.38 & 1.56 \\
\hline
\end{tabular}

\section{Conclusions}

The use of a maple-seed robotic flier (MRF), with its application in very simple to extremely complex multidisciplinary learning, has led to research-oriented and fun-loaded educational modules for $\mathrm{K}-12$, undergraduate and graduate students. The gradual increase of complexity level and open-design concepts allow the learners to develop their own designs and products, and encourage entrepreneurship. Three generations of MRFs were successfully designed, fabricated and tested. The most important considerations for generation 4 are the number of wings and the type of fabrication technology. The evaluation of an undergraduate lab course for freshmen reveals that MRF experiments are well designed so that the innovation and fun within the experiment can catch students' interest and increase their 
intrinsic motivation to engage in the activity which may contribute to a positive learning experience, making the overall experience valuable.

\section{Acknowledgements}

The authors are thankful to He-chuan Cheng for help with Gen-1 MRF construction, Brian Wright and Gregg Mulder for their help with inkjet-printing and accelerometer soldering and Texas Instruments for the donations of MSP 430 kits.

\section{References}

Alfatlawi, M., Abu-Ageel, A., Gardocki, J., Roe, J., Gur, S., \& Aslam, D. M. (2012). "Passive Maple-seed Robotic Fliers (MRF) with On-board Accelerometer \& Microcontroller”, Proc. of IEEE SEM Fall Conf., Dearborn, MI, 2012.

Aslam, D. M. (2006). "Technology Assisted Science, Engineering and Mathematics (TASEM) Education at all Levels Using K-Ph.D. Concept”, Proc. ASEE Annual Conference, Chicago, pp-92, 2006.

Aslam, D. M., \& Shao, A. (2008). "Nanotechnology Learning Modules Using Technology Assisted Science, Engineering and Mathematics", Proc. ASEE Zone 1 Conf., pp., 2008.

Aslam, D. M., \& Wise, K. D. (2003). "Micro and Nano Technologies in K-12", Sensors Expo conference, Session Keynote, June 4, Chicago. http://dx.doi.org/10.1109/TE.2011.2131143

Aslam, D. M., Cao, Z., Varney, M., \& Gur, S. (2010). Outreach and Workforce Training using Maple-Seed Robotic Fliers (MRF), Proc. of IEEE SEM Fall Conf., Dearborn, MI, 2010.

Beck, K., \& Andres, C. (2004). Extreme Programming Explained: Embrace Change (2nd Edition), Publisher: Addison-Wesley Professional. For NXT robot see: http://mindstorms.lego.com/en-us/Default.aspx

Feisel, L., \& Rosa, A. (2005). The role of the laboratory in undergraduate engineering education. Journal of Engineering Education, 94(1), 121-130. http://dx.doi.org/10.1002/j.2168-9830.2005.tb00833.x

Foerster, F., Smeja, M., \& Fahrenberg, J. (1999). Detection of posture and motion by accelerometry: a validation study in ambulatory monitoring. Computers in Human Behavior, 15, 571-583. http://dx.doi.org/10.1016/S0747-5632(99)00037-0

Fong, D. T. W., Wong, J. C. Y., Lam, A. H. F., Lam, R. H.W., \& Li, W. J. (2004). A Wireless Motion Sensing System Using ADXL MEMS Accelerometers for Sports Science Applications. Proceeding of the 5th world congress on intelligent control and Automation, June 15-19.2004. http://dx.doi.org/10.1109/WCICA.2004.1343815

Gianchandani, Y. B. \& Najafi, K. (1992). A bulk silicon dissolved wafer process for microelectromechanical devices. Journal of Microelectromechanical Systems, 1(2), 77-85. http://dx.doi.org/10.1109/84.157361

Harned, W. D. (1997). "Maple-seed Simulating Auto-rotating Toy and Associated Game", US Patent 5,664,783, 1997.

Jameson, S. M., Boesch, B. P., \& Allen, E. H. (2010). “Active Maple Seed Flyer”, US Patent 7,776,274, 2010.

Jimenez Jojoa, E. M., Bravo, E. C., \& Bacca Cortes, E. B. (2010). Tool for Experimenting With Concepts of Mobile Robotics as Applied to Children's Education. Education, IEEE Transactions on , 53(1), 88-95. http://dx.doi.org/10.1109/TE.2009.2024689

Kosuge, K. (2008). "Human-robot interaction - What we learned from robot helpers and dance partner robots", 17th IEEE Int. Symposium on Robot and Human Interactive Communication. http://dx.doi.org/10.1109/ROMAN.2008.4600626

Kovacs, G. T. A., Maluf, N. I., \& Petersen, K. E. (1998). Bulk micromachining of silicon. Proceedings of the IEEE, 86(8), 1536-1551. http://dx.doi.org/10.1109/5.704259

Krajicik, J. S., \& Sutherland, L. M. (2010). Supporting Students in Developing Literacy in Science. Science, 328(5977), 456-459. http://dx.doi.org/10.1126/science.1182593

Olson, M. M., Templeton, L. J., et al. (2007). Production of tyrosine from sucrose or glucose achieved by rapid genetic changes to phenylalanine-producing Escherichia coli strains. Appl Microbiol Biotechnol, 74(5), 1031-40.

Sanchez-Romaguera, V. et al. (2008). Inkjet printing of 3D metal-insulator-metal crossovers. Reactive \& Functional Polymers, 68, 1052-1058. http://dx.doi.org/10.1016/j.reactfunctpolym.2008.02.007

Singh, S. P. N., Fitch, R., \& Williams, B. (2006). “A Research-driven Approach to Undergraduate Robotics Education” Australian Centre for Field Robotics, The University of Sydney NSW, 2006; http://www-personal.acfr.usyd.edu.au/spns/pubcache/CIE_2010_Research_Approach_Education.pdf

Sullivan, F. R. (2008). Robotics and Science Literacy: Thinking Skills, Science Process Skills and Systems Understanding. J. Res. Sci. Teach., 45, 373-394. http://dx.doi.org/10.1002/tea.20238 
Thakoor, S. (2004). "Bio-Inspired Engineering of Exploration Systems"; http://trs-new.jpl.nasa.gov/dspace/handle/2014/16391?mode=full\&submit_simple=Show+full+item+record

Ulrich, E. R. (2010). Control model for robotic samara: Dynamics about a coordinated helical turn, Proc. American Control Conference (Baltimore, MD), 45-50, 2010.

van Osch, T. H. J., Perelaer, J., de Laat, A. W. M., \& Schubert, U. S. (2008). Inkjet Printing of Narrow Conductive Tracks on Untreated Polymeric Substrates. Adv. Mater., 20, 343-345. http://dx.doi.org/10.1002/adma.200701876

Varney, M., Janoudi, A., Aslam, D. M., \& Graham, D. (2012). Building Young Engineers: TASEM for Third Graders in Woodcreek Magnet Elementary School. IEEE Trans. Edu, 99, 2012.

\section{(cc) BY}

This work is licensed under a Creative Commons Attribution 3.0 License. 